\title{
Questioning definitions at university: the case of analysis
}

\section{CARL WINSL $\varnothing W^{1}$}

Résumé. A la base d'un cas particulier, lié à la définition des intégrales curvilignes en analyse vectorielle, nous examinons un dessin modeste visant à réaliser le paradigme du «questionnement du monde » dans l'étude d'un certain type de définition en analyse, et plus généralement les conditions et contraintes rencontrés par ce paradigme dans l'enseignement supérieur des mathématiques.

\begin{abstract}
Based on a case study on task design related to the definition of curve integrals in vector analysis, we examine a modest design for realizing the paradigm of "questioning the world" in the study of a certain type of definition in mathematical analysis, and more generally, the conditions and constraints this paradigm meets in university mathematics education.
\end{abstract}

\section{Definitions in mathematical analysis and elsewhere}

Judging from the average mathematics journal paper or graduate textbook, and the space devoted to proofs, advanced mathematics is all about proving. Definitions, on the other hand, are merely stated and functions as prerequisites for proofs - as necessary, but apparently trivial conventions of language. Occasionally, and especially in less advanced textbook, we find interesting exceptions: a definition is pointed out as important, and its meaning is discussed in terms of examples, technical subtleties and perhaps even the historical genesis of the theory to which it belongs.

From the point of view of ATD, definitions in mathematics could be considered a special instance of a much more general phenomenon: institutions objectify and systematize elements of their technical discourse by developing a specialised superstructure of terminology, assumptions, and shared (within the institution) knowledge about the practice. The language of an institution (e.g. a profession) is full of more or less explicitly defined terms and these are not mere labels: they often constitute objects to which is essential for members of the institution to relate. They are, in themselves, essential works of the institution and at the same time, may be crucial to decide who belongs to it, at least in the sense of necessary conditions: "any member $x$ of $I$ knows what Nanana means to $I$, including what functions Nanana has in $I$, and what $I$ assumes and knows about Nanana". It is evident, then, that definitions in the sense of explicit explanations of institutional terminology may be of specific interest from a didactic point of view, that is, for the process of becoming a member of $I$ through acquiring the practice and knowledge proper to $I$.

In the case of mathematics, we have already mentioned the didactic practice of introducing new objects and properties (or, if you want, "terms") explicitly in the formal setting of a definition, such as:

\footnotetext{
${ }^{1}$ IND, Faculty of Science, University of Copenhagen, Denmark - winslow@ind.ku.dk

El paradigma del cuestionamiento del mundo en la investigación y en la enseñanza

Eje 3. Cuestionar el mundo: avances hacia un nuevo paradigma
} 
Definition 2.1. Let $f: A \rightarrow \mathbb{R}^{m}, A \subset \mathbb{R}^{k}$ and $\mathbf{a} \in \bar{A} \backslash A$ be given and let $\mathbf{b} \in \mathbb{R}^{m}$. One says that $f$ has $\mathbf{b}$ as limit point as $\mathbf{x} \rightarrow \mathbf{a}, \mathbf{x} \in A$ (read: $\mathbf{x}$ goes to $\mathbf{a}$ from $A$ ), and we write $f(\mathbf{x}) \rightarrow \mathbf{b}$ as $\mathbf{x} \rightarrow \mathbf{a}, \mathbf{x} \in A$, if

$$
\forall \varepsilon>0 \exists \delta>0:\|f(\mathbf{x})-\mathbf{b}\|<\varepsilon \text { for all } \mathbf{x} \in A \text { with }\|\mathbf{x}-\mathbf{a}\|<\delta .
$$

(Eilers, Hansen, \& Madsen, 2015, p. 28); translated from Danish by the author, as all other quotes from this source.

The text which accompanies the above definition constitutes, in fact, a rare exception to the general rule that definitions are "merely stated" in mathematics texts. In fact, the definition is located in a section (ibid., pp. 27-38) that contains no theorems or proofs, just this definition and an exposition of its meaning, with both concrete examples and more abstract explanations. There is even an introductory note on its importance and genesis (ibid., p. 27):

A characteristic hallmark of mathematical analysis among other mathematical disciplines is the use of limits. As differential- and integral calculus blossomed from the end of the $17^{\text {th }}$ century, one settled with rather loose ideas, but around 1820, Bernard Bolzano and Augustin-Louis Cauchy succeeded in given the concept a precise meaning. Especially Cauchy's textbook (1821) became an influential breakthrough.

We note in passing that the definition formulated by Cauchy is evidently quite different from the one cited above (Grabiner, 1978). No other definition in the book is given a similar treatment, even if it contains many definitions which are at least as complex as the one cited above.

The textbook above is used in a second semester course on Analysis at the University of Copenhagen. In the 7 weeks the course lasts, students are presented with a large number of definitions, theorems and proofs, beginning with limits and continuity as outlined above, passing through differentiability and integrability in several variables, and ending with vector analysis. At the oral exam, students have to present a "subject", drawn at random among 12 "subjects" such as "The Riemann integral", "Gauss' Theorem" etc. The motivation for this paper is a number of observations, done in the setting of a development project in the spring of 2015, which suggest that definitions may be an overlooked didactical challenge in the course. In particular, we became interested in the following questions: What is the nature of students' difficulties with definitions in first courses on analysis? What relations could or should students develop to definitions? What practices could help students to establish such relations?

We investigate these questions both theoretically and empirically, based on data from our experimentation of new types of student assignments, which can be considered concrete hypotheses for the last question. In section 2, we present a detailed account of a specific case of task design, aiming at helping students relate, in a critical and reflective way, to a complex object of the theory of vector analysis (the definition of curve integrals); we also present some of our observations and analyses of students' work with the task. On this background, Section 3 explain how the case is exemplary of a whole range of definitions found throughout classical analysis, so that in-depth inquiry related to one such definition may in fact have effects on the relationships which students can develop, more broadly, to the theoretical blocks of this domain. Finally, in Section 4, we discuss how promoting such inquiry is aligned with the "paradigm of questioning the world", and how this can be given a specific meaning for the case of higher education in mathematics. 


\section{A case of task design}

In the development project alluded to above, institutional constraints prevented any change in the exam format or the contents of the lectures. Other institutional conditions were more favourable: it is recognised by all teachers that students need better motivation to work with the course material in other ways that merely memorizing proofs for the exam, which leads to highly unsatisfactory results for at least one third of the roughly 300 students who take the course every year. Our topos was the option to partially redesign the exercises worked on in the course and to include these as "supplementary questions" at the oral exam (along with the 12 "main questions"). We designed a total of 57 new exercises, with the aim of enhancing students' autonomous relation to the theoretical blocks in the course, with visible links both to the 12 questions and to students' praxeological equipment from the first semester prerequisite course on calculus.

\subsection{The theoretical context of the exercises}

We now give an example of two such exercises, but first we describe the context: a section in Eilers et al. (2015) entitled "Curve Integral" and composed essentially of one definition and two theorems (both followed by proofs of 1-2 pages). Here is the definition:

Definition 7.20. Let $\Omega \subseteq \mathbb{R}^{k}$ be given. Let $\mathbf{V}: \Omega \rightarrow \mathbb{R}^{k}$ be a vector field in $\Omega$, and let $\gamma$ be a continuous curve in $\Omega$, with parametrization $\mathbf{r}:[a, b] \rightarrow \mathbb{R}^{k}$. We say that the curve integral of $\mathbf{V}$ along $\gamma$ exists, if there is a number $I \in \mathbb{R}$ with the following property: for all $\varepsilon>0$ there is a $\delta>0$ such that for every partition $D$ of $[a, b]$ with fineness $<\delta$ and for every choice of intermediate points, the corresponding middle sum satisfies $\left|I-\sum_{i=1}^{n} \mathbf{V}\left(\mathbf{r}\left(\tau_{i}\right)\right) \cdot \Delta \mathbf{r}_{i}\right|<\varepsilon$. In this case, $I$ is called the curve integral of $\mathbf{V}$ along $\gamma$, and is written $\int_{\gamma} \mathbf{V} \cdot d \mathbf{r}$. (Eilers et al., 2015, p. 230)

The definition is followed by a short remark:

It is easy to see that the curve integral is a geometric object, in the sense that it does not depend on the chosen parametrization. If $\tilde{\mathbf{r}}=\mathbf{r} \circ \varphi$ is a competing parametrization, it gives exactly the same numbers in the middle sum as with $\mathbf{r}$ (Eilers et al., 2015, p. 230).

Finally, the two theorems follow: Theorem 7.21, that $\int_{\gamma} \mathbf{V} \cdot d \mathbf{r}$ exists whenever $\mathbf{V}$ is continuous and $\gamma$ is rectifiable; and Theorem 7.22, that if $\gamma$ admits a $C^{1}$-parametrization $\mathbf{r}$, then $\int_{\gamma} \mathbf{V} \cdot d \mathbf{r}=$ $\int_{a}^{b} \mathbf{V}(\mathbf{r}(t)) \cdot \mathbf{r}^{\prime}(t) d t$.

If the students draw the exam question "Curve Integrals", they will certainly have to prove one or both of the two theorems. In general, students focus their reading on the technical details of the proofs. However, during the observation of exams, we have repeatedly found that low performing students fail because they do not succeed in explaining or using basic definitions. Evidently, the proofs of the two theorems in question depend crucially on Definition 7.20. So, even within the somewhat superficial perspective of the oral examination, it is important to avoid the risk that some students pass quickly over the definition and focus their energy on memorizing the two proofs. But more importantly, without a thorough study of the definition that relates it to students praxeological equipment, the two theorems on "existence" and "computation" will certainly remain, for them, as mysterious as the Stonehenge monument appear to visitors and historians alike. 


\subsection{The tasks}

With the above considerations in mind, we proposed four exercises, of which we shall only consider the last two here:

\section{Exercise F10.3.}

Let $\mathbf{V}$ and $\mathbf{r}$ be as in Definition 7.20.

a) If $\tilde{\mathbf{r}}:[a, b] \rightarrow \mathbb{R}^{k}$ is a direction preserving $C^{1}$-reparametrization of $\mathbf{r}$, show that $\int_{a}^{b} \mathbf{V}(\tilde{\mathbf{r}}(t)) \cdot \tilde{\mathbf{r}}^{\prime}(t) d t=\int_{a}^{b} \mathbf{V}(\mathbf{r}(t)) \cdot \mathbf{r}^{\prime}(t) d t$.

b) Why does a) say that $\int_{\boldsymbol{\gamma}} \mathbf{V} \cdot d \mathbf{r}$ does not depend on the parametrization?

\section{Exercise F10.4.}

a) According to the textbook, one can convince oneself of the uniqueness of the curve integral in Definition 7.20. Show that there does not exist two different $I_{1}$ and $I_{2}$ satisfying the property in Definition 7.20.

b) Compare with F10.3.

c) Read the comment after Definition 7.20 and explain why the curve integral is a geometric object.

\subsection{A priori analysis of the exercises}

Both exercises focus on the point in the comment after Theorem 7.20, namely that curve integrals depend only on the curve - i.e. a set of points in (and of course the vector field), but not on any particular parametrization of the curve, despite the appearance of a parametrization in Definition 7.20.

The first of the two exercises (F10.3) takes the simplest approach to this problem: namely, the formula $\int_{\gamma} \mathbf{V} \cdot d \mathbf{r}=\int_{a}^{b} \mathbf{V}(\mathbf{r}(t)) \cdot \mathbf{r}^{\prime}(t) d t$ which reduces the computation of curve integrals to finding an "ordinary" integral (which the students met already in high school, and which is also treated in the course, a few weeks before, from the theoretical viewpoint of Riemann). The students have encountered this formula as a definition in the first semester calculus course, and have some experience with calculations based on it. This simpler praxeology is in fact likely to be all they knew about curve integrals before this course. For this reason, it is natural to see that the simpler "definition" (even if valid only for the special case of piecewise smooth curves) in fact gives the same number regardless of the choice of $\mathbf{r}$.

Question a) can be solved with a combination of old and new theoretical knowledge: integration by substitution (a technique the students met at high school and again in calculus in the first semester), and the chain rule for vector valued functions which is treated informally in the calculus course, and with all theoretical details in earlier weeks of the present course.

Question b), in fact, is meant to make students aware of the change of perspective in this course: Definition 7.20, not the formula above, is now the "endorsed" way to define curve integrals, and the connection to the formula is established by Theorem 7.22; together with a), this ensures that $\int_{\gamma} \mathbf{V} \cdot d \mathbf{r}$ does not depend on $\mathbf{r}$.

With this in place, the question of course becomes: why all the fuzz with Definition 7.20? Why not simply define the curve integral as in the calculus setting, and save all the work in this section? Exercise F10.4 attempts to ask these questions in a more concrete way.

In a), a technical work with the definition itself is required, and it should be enhanced by students' recent exposure to a similar proof of the uniqueness of the ordinary Riemann integral: 
assuming, as the exercise proposes, that two numbers $I_{1}$ and $I_{2}$ satisfy the definition, we can show that they must be equal by a very similar argument: for any given $\varepsilon>0$ we let $\delta$ be the smallest of those $\delta_{i}$ we get when using the definition on $\varepsilon / 2$ for each of $I_{1}$ and $I_{2}$, and consider a middle sum $M$ for some partition of fineness less than $\delta$; then $\left|I_{1}-M\right|<\varepsilon / 2$ and $\left|I_{2}-M\right|<$ $\varepsilon / 2$, so $\left|I_{1}-I_{2}\right| \leq\left|I_{1}-M\right|+\left|I_{2}-M\right|<\varepsilon$. In fact, in the outline form given here, the two proofs are identical.

In b), students are supposed to formulate themselves that F10.3 and F10.4 give proofs of the same overall result (independence of $\int_{\gamma} \mathbf{V} \cdot d \mathbf{r}$ on parametrization), but also that the setting of F10.4 is more general, since Definition 7.20 does not require that the curve is piecewise $C^{1}$ (but just continuous). This sense of generality of the definition does not have a direct analogy in the case of the ordinary Riemann integral, which is normally defined only on intervals, which are naturally "smooth".

Finally, c) is an occasion to reflect on the geometric meaning of Definition 7.20, the integral can be considered, informally, an "infinite sum" of products of "function values" (here, for the vector field along the curve) by little "increments" in the integration domain (here, secants along the curve). The analogue between the curve integral and the ordinary Riemann integral might also be considered: while the student are very familiar with the idea that the latter defines a "signed area related to the function graph", the geometric meaning of the curve integral will be harder to interpret for them. The introduction of the chapter briefly mentions the physical significance (work of a force along a path). In fact, we also proposed an exercise to develop this point, but it was eventually rejected by the course teachers, as "students do not like to work with physics in mathematics courses" (argument given in a meeting with designers, cited from memory). Geometry poses no such problem and some small sketches showing $\mathbf{V}\left(\mathbf{r}\left(\tau_{i}\right)\right)$ and $\Delta \mathbf{r}_{i}$ at a point of the some two-dimensional curve, may at least relate some of the symbolic expressions in Definition 7.20 to a visual representation. One may even reach an interpretation of $\int_{\gamma} \mathbf{V} \cdot d \mathbf{r}$ as a kind of "signed area of $\mathbf{V}$ along $\gamma$ " if considering the meaning of the vector product as a signed area of the parallelogram spanned by $\mathbf{V}\left(\mathbf{r}\left(\tau_{i}\right)\right)$ and $\Delta \mathbf{r}_{i}$. It is then also intuitively plausible that the integral does not depend on a choice of parametrization.

\subsection{Some observations on student work}

We now present some specific observations of student work on the exercises above, and then more general trends and data concerning the experience as such. The data we refer to were collected by Gravesen and parts of them are presented in her MSc-dissertation (Gravesen, 2015), while the analysis provided here was done subsequently.

The students are supposed to work at home with exercises, prior to the sessions in which they were worked with. During the course, a focus group of 7 students were followed as follows: during the course, all homework done prior to the sessions was collected (originals or photo copies). Two interviews were conducted: the first, four weeks into the course, and the second a few weeks after the final exam.

Three of the seven students did written homework on the two exercises above. A common trait is that they give a detailed answer to a) in both exercises, while they give short and mostly superficial answers to the "follow up" questions. One can speculate that the success with the a)parts is due to the possibility of a retrocognitive approach, as we have seen that these questions can be answered by mimicking arguments which the students have recently been presented for. 
As for F10.3a), they all use the definition of reparametrization to pick a bijection $\varphi$ between the given interval and another interval, such that $\tilde{\mathbf{r}}=\mathbf{r} \circ \varphi$. But then they treat the cases when $\varphi$ is increasing and decreasing, and naturally get only the desired result in the first case; they simply all forget the word "direction preserving" in the exercise formulation. One could speculate that they recently have been presented for a case where it was important to distinguish the two cases, and overlooked that one of them is ruled out by the assumptions here.

There is more variation - and less substance - in the students' productions for the other questions. Here are the "solutions" for F10.3b):

(student 1): No matter what parametrization of $\gamma$ we choose, it gives the same integral according to

Theorem 7.22.

(student 2) If $\varphi$ is increasing (same endpoints)

(student 3) Have just shown $\int_{\boldsymbol{\gamma}} \mathbf{V} d \mathbf{r}$ is the same for a reparametrization.

Here, student 1 is the only one to explicitly point out that Theorem 7.22 is needed to infer the claim, while the other two may consider that the "old" definition from calculus can be used here. Student 2 probably means that the conclusion is correct "if $\varphi$ is increasing" (this is correct) and in fact, the formulation of the exercise is not entirely clear that we only speak of direction preserving reparametrizations in $b$ ); in fact, conventions of exercise formulations would have been better followed if this assumption was stated before both questions. The answer of student 3 is, at best, unclear, as it is incoherent with the answer to a), where two cases were considered and the equality only found in one of them.

All of the students give a satisfactory solution to F10.4a), although one of them (student 2) writes just a few formulae. This student also does not respond to b) and c), while the others respond briefly to $b$ ) and c):

(student 1) b) It would be a terrible contradiction if $\int_{\gamma} \mathbf{V} \cdot d \mathbf{r}$ depended on the parametrization of $\gamma$.

c) We have just seen that $\int_{\gamma} \mathbf{V} \cdot d \mathbf{r}$ does not depend on the chosen parametrization, so it is a geometric property.

(student 3) b) The curve integral is unique and independent of the parametrization. c) Because of the above it makes no sense to talk of the curve integral belonging to $\mathbf{r}$. We talk instead of it along $\gamma$ as it is irrelevant how it moves from $\gamma_{1}$ to $\gamma_{2}$.

It seems obvious that the students have found it difficult to relate to these last questions: to state the difference between the conclusions that can be inferred from F10.3a) and 10.4a) (they merely repeat the rough conclusion given in the exercise formulation, with no regard for the different assumptions and proofs), and they have no firm idea of what "geometric object" could mean. This is quite striking, as the whole point of the section is, in a sense, to investigate the general Definition 7.20. With the calculus approach, there is no question of existence (Theorem 7.21 is superfluous), uniqueness is proved by 10.3a), and Theorem 7.22 is trivial as it is just the definition.

At this point, our readers may object: well, then the whole thing simply was beyond students' grasp. And how about the four students who did not even produce anything before class? To this, two things must be said: first, that one of the motivations for redesigning the exercise material for the course was that in earlier years, course teachers reported that virtually no student ever came to exercise class with any preparation at all, as they found this work less relevant for the oral exam than meticulous reading of the proofs they are to present there. The 
design of exercises closely related to the exam questions was intended to change that and, indeed, the fact that about half the students prepared any given exercise in the present edition of the course, represents a genuine improvement. Moreover, we often found that even students who met without written preparation were able to contribute to solutions in class sessions.

In the present case and in the class we observed, not much was added during the class session to what the students had prepared from home. While the a)-questions were covered in detailed blackboard writing (clarifying also the point about directions in 10.3a), the remaining questions were dealt with quickly, orally and superficially. For instance, here is what one course teacher said after the detailed discussion of 10.4a), to which several students had contributed:

Are there any questions to this? Everything's on track? Perfect. And that's lucky, 'cause what we have shown is then, there is only one curve integral, and the definition also says is therefore, that if that $I$ exists, that was what I wrote here [points at $I$ ], then that $I$ is equal to [writes $\int_{\boldsymbol{\gamma}} \mathbf{V} \cdot d \mathbf{r}$ ] our curve integral. That fits really well with what showed in the exercise right before, so there is only one curve integral. It is precisely the same we show here. There is one and only one, each time we have some curve.

We suspect that the class teachers - who are mostly master students who had a similar course a few years ago - were themselves having difficulties to relate to questions which ask for a deeper reflection on the work accomplished in the initial (and essentially reproductive) reasoning. The instructor's short monologue suggests that those supplementary questions are really trivial and in particular that F10.3 and F10.4 show "precisely" the same.

We note here that the use of students as class instructors is a very solid institutional constraint in Danish universities (at least in science faculties), both for financial reasons and because of its perceived formative functions of having "older" students teach "younger" ones. At the same time, during the experiment we study here, we repeatedly observed it as a challenge in classes when they dealt with exercises requiring non-trivial reflections about course contents.

\section{Definition in analysis: a more general view}

While the exercises above may have left some readers with the impression that this Danish course really deals with rather exotic and technical subtleties of higher mathematics, we shall now demonstrate that they are indeed simply cases of a very general and fundamental challenge which students encounter with definitions in analysis.

In a seminal study of the teaching of the theme "limits of functions" in Spanish high school, Barbé, Bosch, Espinoza, and Gascòn (2005) observed the bipartite character of the praxeologies involved in the students' work with limits of functions at a point, and the difficulties it causes for the organisation of a coherent and meaningful didactic process. In fact, this is a more general problem in the teaching of analysis at secondary level (Winsløw, 2015): from the first study of exponential functions, over the introduction of limits, to the treatment of derivatives and integrals, one rediscovers the gap between algebraic and topological praxeologies. On the one hand, the main aim is to enable students to work concretely with certain objects and transformations (like exponential models), which requires a heavy machinery of algebraic techniques. On the other hand, the mathematical meaning and existence of these objects and transformations requires theory (and so, praxeologies) of a topological nature, and at secondary level these are usually heavily reduced if not entirely omitted from the curriculum. 
Considering the case of limits of a function at a point, a central point noted by Barbé et al. (2005) is that whilst heavy procedures to find limits (by hand or by calculator) may be taught in secondary schools, the questions of conditions of existence is usually bypassed more or less, as are the origins, meanings and uses of limits. More precisely, the definition of limits is not questioned in any way whatsoever, because it is simply not given.

In university courses on analysis (not calculus), a formal definition of convergence of a function $f$ at a point $a$ is certainly given. One formulation has already been given in this paper (Definition 2.1, cited in the introduction). To exhibit the structural similarity with other definitions in analysis, we reformulate it as follows:

(*) $\exists L \in \mathbb{R} \forall \varepsilon>0 \exists \delta>0 \forall x \in] a-\delta, a+\delta[\backslash\{a\}:|f(x)-L|<\varepsilon$

which may of course be explained and represented in many ways. It takes a short argument based on the triangle inequality to see that at most one number $L$ can satisfy the condition

$$
\forall \varepsilon>0 \exists \delta>0 \forall x \in] a-\delta, a+\delta[\backslash\{a\}:|f(x)-L|<\varepsilon
$$

And so, if (*) holds, exactly one number $L$ satisfies (**), and we call it the limit of $f$ at $a$.

Not all of analysis is based directly or indirectly on the notion of limit. Let us consider the crucial notion of the ordinary integral. A function $f:[a, b] \rightarrow \mathbb{R}$ is said to be integrable, if

(*) $\quad \exists I \in \mathbb{R} \forall \varepsilon>0 \exists \delta>0 \forall m \in M_{\delta}(f,[a, b]):|I-m|<\varepsilon$.

and any $I$ satisfying (*) is said to be an integral of $f$ over $[a, b]$. We naturally have to explain the notation $M_{\delta}(f,[a, b])$ : this is the set of middle sums $\sum_{i=1}^{n} f\left(\tau_{i}\right) \cdot\left(x_{i}-x_{i-1}\right)$ of fineness smaller than $\delta$. For a given $\delta$, such a sum corresponds to a choice of numbers $x_{0}, x_{1}, \ldots x_{n}$ satisfying $a=x_{0}<x_{1}<\cdots<x_{n}=b$ and $x_{i}-x_{i-1}<\delta$ for all $i$, and moreover a choice for each $i$ of a middle point $\left.\tau_{i} \in\left[x_{i-1}, x_{i}\right]\right)$.

Notice that $(*)$ has the same overall structure as $(*)$, the difference being essentially that the last quantification is taken over the set $M_{\delta}(f,[a, b])$ instead of an interval defined by $\delta$. Therefore, the proof that there can be at most one $I$ satisfying

$$
\text { (**) } \quad \forall \varepsilon>0 \exists \delta>0 \forall m \in M_{\delta}(f,[a, b]):|I-m|<\varepsilon
$$

is exactly as the proof that there can be at most one $L$ satisfying (**). So, if $f$ is integrable, the integral is unique.

Definitions of other properties and objects in classical analysis have exactly the same form: it is possible to define the property of existence of the object directly, then prove that existence implies uniqueness, and so (indirectly, through that proof) define the object itself. We have seen in detail how that works for limits (and convergence), and for two kinds of integrability (and integral). Further theorems then provide practical means of "computing" the object (in more or less special cases which ensure existence), typically with means that are essentially algebraic. The same theoretical procedure is followed in the context of objects like the many types of derivatives and integrals in vector analysis (of which Definition 7.20 provides one example). In each and every one of these cases, the calculus approach is to avoid - if not simply to ignore the abstract, indirect definition of the central object, and instead introduce the object as the result of some algebraic procedure of computation. This is done at the expense not only of generality, but also of basic meanings (including raison-d'être) of the object, as well as of theoretical control of existence.

One of the eye-catching features of definitions like $\left(^{*}\right)$ and $(*)$ is the sheer complexity. More informal, intuition-appealing formulations are therefore part of common didactic 
transpositions of the corresponding local and regional organisations, while they may not suffice without a more thorough investigation of the problems to which they can be considered an answer.

For instance, the definition of the Riemann integral can be related in various ways to the intuitive notions of area in the plane; the various ways include simply defining the definite integral in terms of area, which in unfortunate unless area has been given a precise definition with other means (which, if precise, would probably be no less complicated than (*) above). It should be noted here that "area" is not considered as problematic by the students; on the contrary, it appears in several domains of the school curriculum where it is not, usually, problematized. Whether a perceptual notion of area as "extension" (or alternatively weight, considering a slight, uniform thickening of the surface) is acceptable notions in mathematics teaching is of course open to debate. On the other hand, at elementary levels, there could be no alternative: analytic definitions in the complex form analysed above are likely to be impracticable. If so, the teaching of this and other concepts of analysis may be faced with additional didactic obstacles, to the extent the raison-d'etre of the analytic definition includes the unsatisfactory nature of the material, perceptive notion, which has previously been used (or even taught explicitly) to students, typically from elementary school. Questioning the new definition - in a sense which will be further elaborated in the final section - thus necessitates a preliminary questioning of solidly anchored praxeological equipment of the student, in particular the theory of area based on visual perception and loosely related computation rules for some special cases (mainly: rectangles, triangles and disks). Since this equipment has been taught and reinforced by institutionally endorsed practices all the way through primary and secondary school, and even in the preliminary calculus course, this questioning represents a highly non-trivial didactic stake: students need to build new praxeologies which are not mere complements or extensions of old ones, but which are to some extent in radical rupture with the old ones. Without this gesture of questioning old praxeologies, the intellectual necessity of a rupture will not appear. The episodes of work with F-exercises, which we considered in Section 2, were evidently not able to establish this necessity, even for the course instructors: students merely seek to mimic standard proofs with little or no idea of what they prove and why.

\section{Questioning Works}

In this final paragraph, three questions will be addressed (in order):

- What exactly do we mean by "questioning" in ATD, and what does or could it mean in the context introduced above (the study of definitions in undergraduate courses on analysis)?

- What conditions and constraint can be identified for the deliberate questioning of definitions in this context, in terms of the levels of didactic co-determination (Chevallard, 2002)?

- To whom is this deliberate questioning important, and why?

\subsection{Definitions of Questioning and Questioning of Definitions}

Chevallard (2012) proposes a vision of public schooling, where "a set of primary questions to be studied at school constitutes the 'core curriculum', and therefore the foundation of the national pact between society and school", and at the same dismisses the use of questions as "a naive 
trick to get students to meet and study works $O$ that the teacher will have determined in advance". There is little said about the likely or necessary character of these "primary questions » in the text. However, we can get a clue from what the author wrote, twenty four years before:

A society is made of works, that is to say human constructions which aim to bring about answers to certain questions, which are the raisons d'être of these works. To enter a society, to socialize there, is to enter into contact with the works that constitute it, and it is to enter into some of them to become authors of them. (Chevallard, 1998) (translated from French by the author).

While this second text also concludes on the importance of taking "live questions" as foundations for new curricula, the first part cited confirms that questions and works are certainly not antagonistic terms, and that it would be absurd to suggest that society could simply decide to found its schooling on questions instead of works. Schooling exists to facilitate the entrance into society (in general, for general schooling) or a society (of a profession, for advanced schooling); and at least to some extent, this entrance is characterized by the "entering into contact" with the works that "constitute" the society. Another reason for the silliness of opposing works and questions is that deep, fruitful questions are themselves human works, and do not arise out of thin air.

To get beyond the mistaken dichotomy between questions and works, we recall an even older proposal from Chevallard (1985): to focus didactical inquiries on the crucial relationship $R_{I}(X, O)$ to be developed, in an institution $I$ and by a study community $X$, with the work $O$. The quote above seems to specify two extremes: mere "contact" with the work, and becoming "authors". It is quite evident that we talk here of a scale with more than two values; and that the presence of relation to the questions to which the work responds is an important parameter to determine where $R_{I}(X, O)$ is located on this scale. Indeed, in this relation, we may find a more or less strong dialectics between questions and answers that are central to the work $O$. This dialectics is discussed in much more detail by Bosch and Winsløw (2015).

The "contact" may indeed be established exclusively through questions or through answers. The "paradigm of visiting monuments" usually is taken to refer to the last extreme, and it is surely commonplace in school approaches to mathematics. This means that $R_{I}(X, O)$ is established solely in terms of superficial contact with results, techniques and routine exercises that are done to confirm, rather than to question. The act of "questioning" a work $O$ - even a small part, like a definition - is then to enrich $R_{I}(X, O)$ by the formulation and study of questions related to the answers. These questions come in several qualities:

- raison d'être questions: what questions does $O$ answer? What motivated or motivates the study of these questions?

- critical questions: Are the answers valid? what warrants does $O$ give for its answers? Are these warrants acceptable?

- perspective questions: Are the answers complete? How does $O$ relate to other works?

Notice that the commonplace sense of "questioning" is quite different: "to question what $X$ say" usually means: to claim that $X$ is wrong. Indeed, we do not take "the paradigm of questioning the world" to refer to a general negative stance towards whatever works or answers we study; instead, we consider it a paradigm of teaching and research in which relations to works are deliberately and explicitly including questionings along the three axes mentioned above. 
Indeed, considering «small works » like $o=$ Definition 7.20 cited above, belonging to some larger work $O$ (here, Eilers et al. (2015)), all of the above kinds of questions make sense and could help to relate $o$ both to the rest of $O$ and, in principal, to other works $O^{\prime}$ as well - whether $R_{I}\left(X, O^{\prime}\right)$ is already established or a first contact is yet to be made. The two F-exercises considered here insist mostly on raison-d'être questions (questioning o): what makes it different, in particular "geometric" as well as more general, than the algebraic (computational) definition of curve integrals known from calculus? As we saw, these questions are not entirely without answers in $O$ and they are proposed in the context because their study could strengthen $R_{I}(X, O)$ in a way that is compatible with other constraints found within $I$. But the search for raison d'être could also naturally question the genesis of these (and other) analytic definitions of integrals (no answer provided in $O$, so other works would be required, and as we saw in section 3, this could enrich if not change $R_{I}\left(X, O^{\prime}\right)$ for works $O^{\prime}$ studied earlier by $X$ ).

One could certainly imagine a questioning also along the other axes as well: in fact, both exercises implicitly assumes students' reading of Theorem 7.21 as warrant of the validity of $o$, in the sense that $o$ agrees with the algebraic definition in the case of $C^{1}$-curves; in fact, the proof of Theorem 7.21 is a main didactic stake of the course. Another kind of validity is explicitly questioned by F10.4(a), asking if (or in fact, "show that") only one number can satisfy the definition. On the other hand, the question of what it does for non- $C^{l}$ cases remains more open, as does the question of how to recognize or display examples of non-integrability of a vector field $\boldsymbol{V}$ along some curve $\gamma$. Finally, while relations to past works (from calculus) could be made more explicit as interpretations of Theorem 7.21, the question of alternatives would lead (or, perhaps, be more naturally posed) to relate $O$ to more advanced works, in which other definitions of (curve) integrals are given - and for these, in fact, the whole array of questions could be considered again, answers compared, etc.

\subsection{Conditions and constraints}

The conditions and constraints of school mathematics and university mathematics are neither the same nor entirely different. In both cases, the society devolves to an institution $I$ - a school, a university - the task of establishing a set of relations $R_{I}(X, O)$ between more or less definite groups $X$ of students to a set $\mathcal{O}$ of more or less definite works $O$, as well as resources considered adequate to accomplish the task. Unlike schools, universities are usually given large autonomy to define $\mathcal{O}$, although for university mathematics one can distinguish at least one more or less explicit constraint: society expects that $\mathcal{O}$ is pertinent to $X$ for the exercise of more or less definite professions which society holds as important. In the case of mathematics programmes, school teaching at various levels is usually implied. For Danish universities, there exists a decree on the "minimal disciplinary requirements" for high school teachers; in mathematics, these include 10 ECTS in calculus and 10 ECTS in analysis (the total requirements specify 90 ECTS which include also 10 ECTS "didactics and science studies", cf. Retsinformation (2006)). All further specification of what goes under these labels (which are roughly "domains") is left to universities.

At the same time, Danish university programmes in pure mathematics are not "profession oriented" in the sense that they lead to a specific profession; and in fact, only a minority of the graduates take up teaching positions in high schools; a larger group is employed in private companies, and others continue with doctoral studies. The last group ends up in positions both 
inside and outside academia. Those who pursue academic careers contribute to renew the population of university faculty in mathematics. That this is also rather explicitly required by society can be seen from the incitements it provides for doctoral study programmes.

This resumes, in gross outline, the specific conditions and constraints which society imposes university mathematics programmes in more or less specific ways. Naturally, in the case of public universities at least, society imparts other regulations and resources to universities, which can weigh on a specific didactic system like the course we study here. For instance, in Denmark the allocation of resources to universities depends directly on a number of variables, one of which is the number of students who pass exams for individual courses ("the production of ECTS credits"). This, in practice, exercises some pressure on universities to "get students through".

Universities have some freedom to make decisions at the level of Pedagogy (for instance, how teaching is organized and how exams are conducted), where considerable differences exist. This also holds true when it comes to how Disciplines are organized internally and externally; at the University of Copenhagen, both levels can be described as relatively "classical", and at least as far as Mathematics is concerned, ambitious in terms of the relative size of $\mathcal{O}$ and, as timewise length is fixed to $3+2$ years, consequently the relative pace at which the study of $\mathcal{O}$ must be completed.

I think Analysis 0 - which covers part of the Domain "Analysis" - could be an illustration of these rough points. Within seven weeks (where the students devote half of their study time to this course), the students get to study the theory of derivatives and integrals in one and several variables, as well as the basics of vector analysis, at the abstract level exemplified in our case above. The density of themes which the institution prescribes, certainly leaves limited room for questioning definitions, theorems and proofs in the sense discussed in this paper, and the approach to mathematical works is not free of monumentalism in the sense of Chevallard (2004). Only towards the end of the programmes do student get to choose and search works more freely, and in many cases, even the final "Thesis" is quite heavily directed by a supervisor.

\subsection{To whom is questioning important and why?}

Before dismissing this kind of programme as a superficial and anachronistic tour of monuments, let us pause to look, after all, at what Chevallard $(2012$, p. 4) calls "the happy few, who need very little attention": those who become academic mathematicians. For a contemporary university, both external prestige and internal renewal is linked to the education of "top level scientists". There is no shortage of examples of excellent mathematicians coming out of programmes of the "classical type" just described. Indeed, to enter the micro-society (or institution) of mathematicians, one needs to make contact with works from current mathematical research, and subsequently get to question it to the point of contributing to such works. A very long sequence of works needs to be studied to get from didactic texts such as (Eilers et al., 2015) to current works like (Ando, Haagerup, \& Winsløw, 2016). This, at least, is the kind of reason which the institution would give for the size of $\mathcal{O}$ to be studied in five years, and in particular for the size of $O$ to be studied in the first half of the second semester. Other explanations come from internal constraints in the discipline and the way it is organized in the institution. For instance, the sectors and themes studied in Analysis 0 are required for the sectors and themes studied in Analysis 1 and 2, and so on. 
The constraints coming from the aim to provide access to contemporary works of pure mathematics are most evidently justified for the case of students who aim to become research mathematicians. But it is a classical assumption, eloquently supported by prominent figures like Felix Klein (Winsløw \& Grønbæk, 2014), that it could also be meaningful for future teachers.

One can in fact even argue for the societal and intellectual value of the complete visit of $\mathcal{O}$ : to the extent it is really an up-to-date version of those works that "constitute" the society of academic mathematics, "entering in to contact with $\mathcal{O}$ " is a condition sine-qua-non for entering that society and to contribute to it. If so, establishing $R_{I}(X, \mathcal{O})$ in the sense of "contact" or "visits" would be a necessary condition for $X$ being the "happy few". And for some of them, $R_{I}(X, \mathcal{O})$ in fact turns out to become more than minimal, in the sense that they actually get to question recent works and produce new works. For this group at least, the current low emphasis on questioning apparently suffices.

The motivation for the development of didactic infrastructures - such as F-exercises - and therefore also paradidactic infrastructures (Winsløw, 2012) to construct and develop didactic infrastructures - is that a large majority of students do not become professional mathematicians and, under the present regime, leave their studies with (at best) a passing knowledge of mathematics as a beautiful, endless structure of definitions, theorems and proofs, coming out of nowhere and leading mainly to a diploma and then some more or less enjoyable occupation. For example, Winsløw and Grønbæk (2014) studied the relationship $R_{U}(\sigma, \omega)$ of a group of university students $\sigma$ to specific works $\omega \in \mathcal{O}$ and their difficulties, in some case outright impossibilities, to draw on $R_{U}(\sigma, \omega)$ to question definitions (for instance, of the exponential function) at the level of high school.

The questioning of fundamental definitions and other works in analysis remain important for these students as well, if they are to become part of the society which contributes to the works studied at high school. Both schools and other institutions in society need innovative mathematicians, capable of questioning mathematical works and adapting them to concrete purposes. For this reason, and based on documented observation of the insufficiency of current didactic infrastructure, concrete efforts like the F-exercises need to be developed, experimented and assessed - in lucid consideration of the evident and hidden constraints, as well as of the conditions which could support it. The lucidity implies, among other things, a realistic view of what could be obtained on a short and long term, without which the didactician risks being left in a bubble of comfortable utopia, but stripped of all relevance to the practice it wishes to overthrow.

In the case we considered, a first didactic infrastructure has been developed with the design of F-exercises, by making use of favourable conditions offered by the institution to further "research like" activity of students, interpreted here as local questioning of definitions, theorems and proofs. Even to succeed with this modest design, more is needed: a paradidactic infrastructure for the alignment of teaching assistants', lecturer's and examiners' practices both with each other, and with the aim of taking students at least a step beyond the "visiting" of the works of the course. Moreover, as the teaching assistants' relation to the same works are to a large extent the result of their own experiences from the course, one should not underestimate the self-enforcing function of the fact that future instructors will have experienced the new didactic infrastructure as students. 


\section{References}

Ando, H., Haagerup, U., \& Winsløw, C. (2016). Ultraproducts, QWEP von Neumann algebras, and the Effros-Maréchal topology. Journal fuer die Reine und Angewandte Mathematik, 715, 231-250. DOI: 10.1515/crelle-2014-0005

Barbé, J., Bosch, M., Espinoza, L., \& Gascòn, J. (2005). Didactic Restrictions on the Teacher's Practice: The Case of Limits of Functions in Spanish High Schools. Educational Studies in Mathematics, 59(1-3), 235-268.

Bosch, M., \& Winsløw, C. (2015). Linking problem solving and learning contents: the challenge of self-sustained study and research processes. Recherches en Didactique des Mathématiques, 35(2).

Chevallard, Y. (1985). La Transposition didactique: du savoir savant au savoir enseigné. Grenoble: La Pensée Sauvage.

Chevallard, Y. (1998). Questions vives, savoirs moribonds : le problème curriculaire aujourd'hui. Paper presented at the Défendre et transformer l'école pour tous, Marseille. http://yves.chevallard.free.fr/spip/spip/article.php3?id_article=19

Chevallard, Y. (2002). Organiser l'étude 3. Écologie \& régulation. Paper presented at the 11 ème école de didactique des mathématiques.

Chevallard, Y. (2004). Vers une didactique de la codisciplinarité. Notes sur une nouvelle épistémologie scolaire. Paper presented at the Journées de didactique comparée, Lyon. http://yves.chevallard.free.fr/spip/spip/article.php3?id_article=45

Chevallard, Y. (2012). Teaching mathematics in tomorrow's society: a case for an oncoming counterparadigm. Paper presented at the 12th International Congress on Mathematical Education, Seoul. retrieved from http://www.icme12.org/upload/submission/1985 F.pdf

Eilers, S., Hansen, E., \& Madsen, T. G. (2015). Indledende Matematisk Analyse. Copenhagen: University of Copenhagen.

Grabiner, J. (1978). The origins of Cauchy's theory of the derivative. . Historia Mathematica, 5, 379-409.

Gravesen, K. (2015). Forskningslignende situationer på et førsteårskursus $i$ matematisk analyse. (M.Sc.), University of Copenhagen, Copenhagen.

Retsinformation. (2006). Retningslinier for universitetsuddannelser rettet mod undervisning i de gymnasiale udannelser (faglige mindstekrav). https://www.retsinformation.dk/Forms/R0710.aspx?id=29265.

Winsløw C. (2012) A Comparative Perspective on Teacher Collaboration: The Cases of Lesson Study in Japan and of Multidisciplinary Teaching in Denmark. In G. Gueudet, B. Pepin, and L. Trouche (eds), From Text to 'Lived' Resources. Mathematics Teacher Education, vol 7. Dordrecht: Springer.

Winsløw, C. (2015). Mathematical analysis in high school: a fundamental dilemma. In C. Bergsten and B. Sriraman (Ed.), Refractions of Mathematics Education: Festschrift for Eva Jablonka (pp. 197-213). Charlotte, NC: Information Age Publ.

Winsløw, C., \& Grønbæk, N. (2014). Klein's double discontinuity revisited: contemporary challenges for universities preparing teachers to teach calculus. Recherches en Didactique des Mathématiques, 34(1), 59-86. 